\title{
PARETO OPTIMAL RISK EXCHANGES AND A SYSTEM \\ OF DIFFERENTIAL EQUATIONS : A DUALITY THEOREM
}

\author{
BY ERICH WyLER
}

ETH Zürich, Switzerland

\begin{abstract}
This article, based on a result of BORCH and an extension of BÖHLMANN, gives a complete characterization of Pareto optimal risk exchanges by a system of differential equations linking the derivate of agents contributions to their risk aversion coefficients.
\end{abstract}

\section{KEYWORDS}

Pareto optimal risk exchange; Bernoulli utility function; absolute risk aversion; system of differential equations.

\section{INTRODUCTION}

This article extends a result of BÜHLMANN (1984). Starting from BoRCH's theorem (1960), BüHLMANN found a system of differential equations with a Pareto optimal risk exchange as the solution. Here we are starting from these differential equations and prove existence and uniqueness of a solution without assuming any further condition. This solution depends on initial values which satisfy a certain clearing condition. It will turn out that it can be identified in a bijective way with the set of Pareto optimal risk exchanges.

\section{MODEL}

We consider a risk pool with $n$ participants. Participant $i(1 \leq i \leq n)$ is characterized by

$r_{i}$ : initial wealth

$X_{i}$ : initial risk (random variable defined on a probability space $(\Omega, \mathfrak{H}, P)$; we assume that the expected values $E\left[X_{i}\right]$ exists)

$u_{i}$ : Bernoulli utility function (defined on $\mathbb{R}$, increasing, strictly concave and twice differentiable: $\left.u_{i}^{\prime}<0, u_{i}^{\prime \prime}>0\right)$

$\rho_{i}:$ absolute risk aversion $\left(\rho_{i}:=-u_{i}^{\prime \prime} / u_{i}^{\prime}\right.$. Notice $u_{i}^{\prime}>0$ and $\rho_{i}>0$ i.e. the participants are risk averse; see PraTt (1964)).

By a risk pool we mean any formal mutual agreement among the $n$ participants to redistribute their total initial risk $\sum_{i=1}^{n} X_{i}$.

ASTIN BULLETIN, Vol. 20, No. 1 
The intial risk vector $\boldsymbol{X}$,

$$
X:=\left(X_{1}, \ldots, X_{n}\right),
$$

is called risk vector before exchange whereas a risk vector $\boldsymbol{Y}$,

$$
\boldsymbol{Y}:=\left(Y_{1}, \ldots, Y_{n}\right)
$$

defined on the same probability space $(\Omega, \mathfrak{A}, P)$ and satisfying the clearing condition

$$
\sum_{i=1}^{n} Y_{i}=\sum_{i=1}^{n} X_{i}
$$

is denoted as risk vector after exchange or briefly as risk exchange.

Furthermore a risk exchange $Y^{*}:=\left(Y_{1}^{*}, \ldots, Y_{n}^{*}\right)$ is called Pareto optimal if there does not exist another risk exchange $Y:=\left(Y_{1}, \ldots, Y_{n}\right)$ with

$$
\begin{gathered}
E\left[u_{i}\left(r_{i}-Y_{i}^{*}\right)\right] \leq E\left[u_{i}\left(r_{i}-Y_{i}\right)\right] \quad \text { for all } i \\
E\left[u_{i^{0}}\left(r_{i^{0}}-Y_{i^{\circ}}^{*}\right)\right]<E\left[u_{i^{0}}\left(r_{i^{o}}-Y_{i^{\circ}}\right)\right] \quad \text { for at least one } i^{o} .
\end{gathered}
$$

In the sequel we are interested in Pareto optimal risk exchanges.

REMARK. The motivation of a person for participating in a risk pool is to improve his initial expected utility $E[u(r-X)]$. Therefore a risk exchange $Y$ has to satisfy the individual rationality condition

$$
E\left[u_{i}\left(r_{i}-X_{i}\right)\right] \leq E\left[u_{i}\left(r_{i}-Y_{i}\right)\right] \quad \text { for all } i
$$

in addition to the pool condition of Pareto optimality. Unfortunately there are many Pareto optimal risk exchanges violating this condition. In order to preserve the beauty of the main result we drop the individual rationality condition and deal in this article with general Pareto optimal risk exchanges.

In order to simplify our notation we introduce the shifted disutility functions $v_{i}$

$$
v_{i}(x):=u_{i}\left(r_{i}-x\right) \quad i=1, \ldots, n . \quad\left(v_{i}^{\prime}<0, v_{i}^{\prime \prime}<0\right)
$$

With $W_{i}$ we denote the range of the derivative of $v_{i}$

$$
W_{i}:=\left\{v_{i}^{\prime}(x) \mid x \in \mathbb{R}\right\} .
$$

\section{MAIN RESULT}

Now we show the existence of a bijective mapping between the set of Pareto optimal risk exchanges and the set of solutions of a system of differential equations satisfying a constrained boundary condition. 


\section{THEOREM}

Let $\boldsymbol{w}, \boldsymbol{w}:=\left(w_{1}, \ldots, w_{n}\right) \in \mathbb{R}^{n}$, be a vector with $\sum_{i=1}^{n} w_{i}=0$.

(i) Let (A) be the system of differential equations

(A) $\quad Y_{i}^{\prime}(z)=\frac{\overline{\rho_{i}\left(r_{i}-Y_{i}(z)\right)}}{\sum_{j=1}^{n} \frac{1}{\rho_{j}\left(r_{j}-Y_{j}(z)\right)}} \quad i=1, \ldots, n$.

There exists a uniquely defined solution $Y(z)=\left(Y_{1}(z), \ldots, Y_{n}(z)\right)$ of (A) satisfying the boundary condition $Y_{i}(0)=w_{i}, i=1, \ldots, n$.

(ii) If $\boldsymbol{Y}(z)=\left(Y_{1}(z), \ldots, Y_{n}(z)\right)$ is the solution of (A) with boundary condition $Y_{i}(0)=w_{i}, i=1, \ldots, n$, then

$$
\boldsymbol{Y}\left(\sum_{i=1}^{n} X_{i}\right)
$$

is a Pareto optimal risk exchange.

(iii) If $Y^{*}:=\left(Y_{1}^{*}, \ldots, Y_{n}^{*}\right)$ is a Pareto optimal risk exchange then there exists a solution $Y(z)=\left(Y_{1}(z), \ldots, Y_{n}(z)\right)$ of (A) satisfying a uniquely defined boundary condition $Y_{i}(0)=w_{i}, i=1, \ldots, n, \sum_{i=1}^{n} w_{i}=0$, with

$$
\boldsymbol{Y}^{*}=\boldsymbol{Y}\left(\sum_{i=1}^{n} X_{i}\right) \quad \text { almost surely. }
$$

\section{Proof}

(i) Existence of a solution :

Let $f_{k}$ be the function $f_{k}(x):=\sum_{i=1}^{n}\left(v_{i}^{\prime}\right)^{-1}\left(\frac{x}{k_{i}}\right)$ with $k:=\left(k_{1}, \ldots, k_{n}\right)$, $k_{i}:=\frac{-1}{v_{i}^{\prime}\left(w_{i}\right)}>0 . f_{k}$ is a strictly decreasing and differentiable function defined on $W$

$$
W:=\bigcap_{i=1}^{n}\left\{x k_{i} \mid x \in W_{i}\right\}
$$

with range $\mathbb{R}$. (see Lemma 1 , Appendix). Furthermore $f_{k}(-1)=0$. (see Proof of Lemma 1, Appendix). We have

$\boldsymbol{Y}(z):=\left(Y_{1}(z), \ldots, Y_{n}(z)\right)$ with $Y_{i}(z)=\left(v_{i}^{\prime}\right)^{-1}\left(\frac{1}{k_{i}}\left(f_{k}\right)^{-1}(z)\right), i=1, \ldots, n$, 
and $k_{i}:=\frac{-1}{v_{i}^{\prime}\left(w_{i}\right)}, \quad i=1, \ldots, n$, is a solution of (A).

Uniqueness of the solution:

Let $\tilde{\boldsymbol{Y}}(z)=\left(\tilde{Y}_{1}(z), \ldots, \tilde{Y}_{n}(z)\right)$ be another solution of (A) satisfying the same boundary condition. We define differentiable functions $g_{i}(z), i=2, \ldots, n$ :

$$
g_{i}(z):=k_{1} v_{1}^{\prime}\left(\tilde{Y}_{1}(z)\right)-k_{i} v_{i}^{\prime}\left(\tilde{Y}_{i}(z)\right), \quad z \in \mathbb{R} .
$$

We have $g_{i}(0)=0$ for all $i$ and for the derivatives $g_{i}^{\prime}(z), i=2, \ldots, n$, we get

$$
\begin{aligned}
g_{i}^{\prime}(z)= & k_{1} v_{1}^{\prime \prime}\left(\tilde{Y}_{1}(z)\right) \tilde{Y}_{1}^{\prime}(z)-k_{i} v_{i}^{\prime \prime}\left(\tilde{Y}_{i}(z)\right) \tilde{Y}_{i}^{\prime}(z) \\
= & \frac{\frac{k_{1} v_{1}^{\prime \prime}\left(\tilde{Y}_{1}(z)\right)}{\rho_{1}\left(r_{1}-\tilde{Y}_{1}(z)\right)}-\frac{k_{i} v_{i}^{\prime \prime}\left(\tilde{Y}_{i}(z)\right)}{\rho_{i}\left(r_{i}-\tilde{Y}_{i}(z)\right)}}{\sum_{j=1}^{n} \frac{1}{\rho_{j}\left(r_{j}-\tilde{Y}_{j}(z)\right)}} \quad \text { (with (A)) } \\
= & \frac{g_{i}(z)}{\sum_{j=1}^{n} \frac{1}{\rho_{j}\left(r_{j}-\tilde{Y}_{j}(z)\right)}} \quad z \in \mathbb{R} .
\end{aligned}
$$

Because the homogeneous linear differential equations

$$
g_{i}^{\prime}(z)=\frac{g_{i}(z)}{\sum_{j=1}^{n} \frac{1}{\rho_{j}\left(r_{j}-\tilde{Y}_{j}(z)\right)}}, \quad z \in \mathbb{R}, \quad i=2, \ldots, n
$$

have only solutions of the form

$$
g_{i}(z)=c_{i} \exp \left(\int_{0}^{z} \frac{1}{\sum_{j=1}^{n} \frac{1}{\rho_{j}\left(r_{j}-\tilde{Y}_{j}(t)\right)}} d t\right), \quad c_{i} \in \mathbb{R}, \quad i=2, \ldots, n
$$

we get together with $g_{i}(0)=0: c_{i}=0$ and therefore $g_{i}(z)=0$ for all $z \in \mathbb{R}$ and $i=2, \ldots, n$.

This means

$$
k_{i} v_{i}^{\prime}\left(\tilde{Y}_{i}(z)\right)=k_{1} v_{1}^{\prime}\left(\tilde{Y}_{1}(z)\right) \text { for all } z \in \mathbb{R} \text { and } i=2, \ldots, n .
$$

Because $\sum_{i=1}^{n} \tilde{Y}_{i}^{\prime}(z)=1$ for all $z \in \mathbb{R}$ it follows together with the boundary condition that $\sum_{i=1}^{n} \tilde{Y}_{i}(z)=z$ for all $z \in \mathbb{R}$. 
Because $\tilde{Y}(z)$ and $\boldsymbol{Y}(z)$ satisfy both the equations $\left(^{* *}\right)$ of Lemma 2 (see Appendix) we conclude by uniqueness of the solution that

$$
\tilde{Y}(z)=\boldsymbol{Y}(z) \quad \text { for all } \quad z \in \mathbb{R} \text {. }
$$

(ii) $Y(z):=\left(Y_{1}(z), \ldots, Y_{n}(z)\right)$ with $Y_{i}(z)=\left(v_{i}^{\prime}\right)^{-1}\left(\frac{1}{k_{i}}\left(f_{k}\right)^{-1}(z)\right), i=1, \ldots, n$, and $k_{i}:=\frac{-1}{v_{i}^{\prime}\left(w_{i}\right)}, i=1, \ldots, n$, is the unique solution of (A)

satisfying the boundary condition $Y_{i}(0)=w_{i}, i=1, \ldots, n$. Because this solution satisfies $\left({ }^{* *}\right)$ of Lemma 2, (see Appendix), it follows from BorCH's theorem (see Borch, (1960)) that $Y\left(\Sigma X_{i}\right)$ is a Pareto optimal risk exchange.

(iii) It follows from BORCH's theorem (see BoRCH, (1960)) that there are strictly positive constants $k_{i}, i=1, \ldots, n$, with

$$
k_{i} v_{i}^{\prime}\left(Y_{i}^{*}\right)=k_{1} v_{1}^{\prime}\left(Y_{1}^{*}\right) \text { almost surely for } i=2, \ldots, n \text {. }
$$

Let $\omega \in \Omega$ be an element of $\Omega$ for which the condition of BoRCH is satisfied, $k$ the vector $\boldsymbol{k}:=\left(k_{1}, \ldots, k_{n}\right)$ and $f_{k}$ the function as defined above. Because $f_{k}(x)$ is defined for $x:=k_{1} v_{1}^{\prime}\left(Y_{1}^{*}(\omega)\right)$

$$
f_{k}(x)=\sum_{i=1}^{n}\left(v_{i}^{\prime}\right)^{-1}\left(\frac{x}{k_{i}}\right)=\sum_{i=1}^{n}\left(v_{i}^{\prime}\right)^{-1}\left(\frac{k_{i} v_{i}^{\prime}\left(Y_{i}^{*}(\omega)\right)}{k_{i}}\right)=\sum_{i=1}^{n} Y_{i}^{*}(\omega)
$$

(condition of BORCH)

it follows analogously to Lemma 1 , (see Appendix), that $f_{k}$ is defined on some interval $(a, b)$ with range $\mathbb{R}$. Therefore $\left(f_{k}\right)^{-1}(0)$ exists. We define the vector $w=\left(w_{1}, \ldots, w_{n}\right)$ by

$$
w_{i}:=\left(v_{i}^{\prime}\right)^{-1}\left(\frac{1}{k_{i}}\left(f_{k}\right)^{-1}(0)\right), \quad i=1, \ldots, n .
$$

The unique solution $Y(z)=\left(Y_{1}(z), \ldots, Y_{n}(z)\right)$ of (A) with boundary condition $Y_{i}(0)=w_{i}, i=1, \ldots, n$, satisfies the equations $\left({ }^{*}\right)$ of Lemma 2, i.e.

$$
\begin{aligned}
& \sum_{i=1}^{n} Y_{i}(z)=z \quad \text { for all } \quad z \in \mathbb{R} \\
& k_{i} v_{i}^{\prime}\left(Y_{i}(z)\right)=k_{1} v_{1}^{\prime}\left(Y_{1}(z)\right) \text { for } i=2, \ldots, n \text { and all } z \in \mathbb{R} .
\end{aligned}
$$

We conclude by uniqueness of the solution that

$$
Y^{*}=Y\left(\sum_{i=1}^{n} X_{i}\right) \quad \text { almost surely }
$$


REMARK: Because $-1 / k_{i}$ is possibly not in the range of $v_{i}^{\prime}$ we cannot

define $w_{i}$ by $\left.w_{i}:=\left(v_{i}^{\prime}\right)^{-1}\left(\frac{-1}{k_{i}}\right)\right)$.

QED

4. EXAMPLE

We assume that the participants are using exponential utility functions, i.e. $\rho_{i}(x)=a_{i}$ for all $x \in \mathbb{R}$ and $i=1, \ldots, n$, where $\rho_{i}$ denotes the absolute risk aversion of participant $i$. In this case the system of differential equations (A) becomes very simple

$$
\text { (A) } \quad Y_{i}^{\prime}(z)=\frac{\frac{1}{a_{i}}}{\sum_{j=1}^{n} \frac{1}{a_{j}}}, \quad i=1, \ldots, n .
$$

We therefore have

$$
Y_{i}(z)=\frac{\frac{1}{a_{i}}}{\sum_{j=1}^{n} \frac{1}{a_{j}}} z+\beta_{i}, \quad i=1, \ldots, n,
$$

where the $\beta_{i}$ 's satisfy the clearing condition

$$
\sum_{i=1}^{n} \beta_{i}=0
$$

For further examples, e.g. for utility functions of the HARA-type, see LIENHARD, (1986).

\section{APPENDIX}

To conclude the two technical lemmas already used in the proof of the main Theorem are discussed.

LEMMA 1

Let $\quad \boldsymbol{w}, \boldsymbol{w}:=\left(w_{1}, \ldots, w_{n}\right) \in \mathbb{R}^{n}$, be a vector with $\sum_{i=1}^{n} w_{i}=0$

and $\boldsymbol{k}, \boldsymbol{k}:=\left(k_{1}, \ldots, k_{n}\right) \in \mathbb{R}^{n}$ the vector with $k_{i}:=\frac{-1}{v_{i}^{\prime}\left(w_{i}\right)}>0$. 
Then $f_{k}(x):=\sum_{i=1}^{n}\left(v_{i}^{\prime}\right)^{-1}\left(\frac{x}{k_{i}}\right)$ is a strictly decreasing and differentiable

function defined on $W$

$$
W:=\bigcap_{i=1}^{n}\left\{x k_{i} \mid x \in W_{i}\right\}
$$

with range $\mathbb{R}$.

\section{Proof}

Obviously $W$ is an open interval. $W$ is not empty because it contains -1 .

$$
f_{k}(-1)=\sum_{i=1}^{n}\left(v_{i}^{\prime}\right)^{-1}\left(\frac{-1}{k_{i}}\right)=\sum_{i=1}^{n}\left(v_{i}^{\prime}\right)^{-1}\left(v_{i}^{\prime}\left(w_{i}\right)\right)=\sum_{i=1}^{n} w_{i}=0 .
$$

We denote by $\left(a_{i}, b_{i}\right)$ the open interval $W_{i}$ and by $(a, b)$ that of $W$. We have $a=a_{i} k_{i} \quad$ for at least one $\quad i$

and therefore

$\lim _{x \rightarrow a} f_{k}(x)=\lim _{x \rightarrow a} \sum_{i=1}^{n}\left(v_{i}^{\prime}\right)^{-1}\left(\frac{x}{k_{i}}\right) \geq \lim _{\substack{x \rightarrow a_{i} k_{i} \\ x>a}}\left(v_{i}^{\prime}\right)^{-1}\left(\frac{x}{k_{i}}\right)=\lim _{\substack{y \rightarrow a_{i} \\ y>a_{i}}}\left(v_{i}^{\prime}\right)^{-1}(y)=\infty$.

Analoguously we get

$$
\lim _{x \rightarrow b} f_{k}(x)=-\infty
$$

It follows that the continuous function $f_{k}$ has range $\mathbb{R}$. Obviously $f_{k}$ is differentiable on $W$ with derivative

$$
f_{k}^{\prime}(x)=\sum_{i=1}^{n} \frac{1}{k_{i}} \frac{1}{v_{i}^{\prime \prime}\left(\left(v_{i}^{\prime}\right)^{-1}\left(\frac{x}{k_{i}}\right)\right)}<0 .
$$

\section{LEMMA 2}

Let $\quad \boldsymbol{w}, \boldsymbol{w}:=\left(w_{1}, \ldots, w_{n}\right) \in \mathbb{R}^{n}$, be a vector with $\sum_{i=1}^{n} w_{i}=0$

and $\boldsymbol{k}, \boldsymbol{k}:=\left(k_{1}, \ldots, k_{n}\right) \in \mathbb{R}^{n}$ the vector with $k_{i}:=\frac{-1}{v_{i}^{\prime}\left(w_{i}\right)}>0$.

Furthermore let $\left(^{*}\right)$ and $\left({ }^{* *}\right)$ respectively denote the system of equations (in $\left.Y_{1}(z), \ldots, Y_{n}(z)\right)$ 


$$
z=f_{k}\left(k_{i} v_{i}^{\prime}\left(Y_{i}(z)\right)\right) \quad i=1, \ldots, n .
$$

$(* *) \quad\left\{\begin{array}{l}\sum_{i=1}^{n} Y_{i}(z)=z \quad \text { for all } \quad z \in \mathbb{R} \\ k_{i} v_{i}^{\prime}\left(Y_{i}(z)\right)=k_{1} v_{1}^{\prime}\left(Y_{1}(z)\right) \text { for } i=2, \ldots, n .\end{array}\right.$

Then $Y(z):=\left(Y_{1}(z), \ldots, Y_{n}(z)\right)$ with $Y_{i}(z)=\left(v_{i}^{\prime}\right)^{-1}\left(\frac{1}{k_{i}}\left(f_{k}\right)^{-1}(z)\right), i=1, \ldots, n$,

is the unique solution of $\left(^{*}\right)$ resp. $\left({ }^{* *}\right)$. The functions $Y_{i}(z), i=1, \ldots, n$, are strictly increasing and differentiable. They satisfy $Y_{i}(0)=w_{i}$ for $i=1, \ldots, n$.

\section{Proof}

From Lemma 1 it follows that $\left(f_{k}\right)^{-1}$ exists and is defined on $\mathbb{R}$. Therefore $\boldsymbol{Y}(z)$ is well defined, strictly increasing and differentiable. By inverting equation $\left(^{*}\right)$ we see that $Y(z)$ is a solution and even the unique solution of $\left(^{*}\right)$. Obviously $Y(z)$ is also a solution of $(* *)$.

Note that

$$
\begin{aligned}
& \sum_{i=1}^{n} Y_{i}(z)=f_{k}\left(\left(f_{k}\right)^{-1}(z)\right)=z \\
& k_{i} v_{i}^{\prime}\left(Y_{i}(z)\right)=\left(f_{k}\right)^{-1}(z)=k_{1} v_{1}^{\prime}\left(Y_{1}(z)\right) \text { for } i=2, \ldots, n .
\end{aligned}
$$

Furthermore

$$
Y_{i}(0)=\left(v_{i}^{\prime}\right)^{-1}\left(\frac{1}{k_{i}}(-1)\right)=w_{i} \quad(\text { see proof of Lemma } 1)
$$

Let $\tilde{Y}(z):=\left(\tilde{Y}_{1}(z), \ldots, \tilde{Y}_{n}(z)\right)$ be another solution of $\left(^{* *}\right)$. Then we have

$$
\begin{aligned}
z=\sum_{i=1}^{n} \tilde{Y}_{i}(z) & =\sum_{i=1}^{n}\left(v_{i}^{\prime}\right)^{-1}\left(\frac{k_{1}}{k_{i}} v_{1}^{\prime}\left(\tilde{Y}_{1}(z)\right)\right) \\
& =f_{k}\left(k_{1} v_{1}^{\prime}\left(\tilde{Y}_{1}(z)\right)\right)=f_{k}\left(k_{i} v_{i}^{\prime}\left(\tilde{Y}_{i}(z)\right)\right)
\end{aligned}
$$

But the solution of (*) is unique, so we have $\overline{\boldsymbol{Y}}(z)=\boldsymbol{Y}(z)$ for all $z \in \mathbb{R}$. This completes the proof.

QED 


\section{REFERENCES}

Borch, K. (1960) The Safety Loading of Reinsurance Premiums. Skandinavisk Aktuarictidskrift 43, 163-184.

Bohlmann, H. (1980) An Economic Premium Principle. ASTIN Bulletin 11, 52-60.

Búhlmann, H. (1984) The General Economic Premium Principle. ASTIN Bulletin 15, 13-21.

Lienhard, M. (1986) Calculation of Price Equilibria for Utility Functions of the HARA class. ASTIN Bulletin 17, 91-97.

PratT, J.W. (1964) Risk Aversion in the Small and in the Large. Econometrica 32, 122-136.

\section{ERICH WyLER}

Anna-Heer-Strasse 28, CH-8057 Zürich, Switzerland. 